\title{
An Alarming Increase of Fungal Infections in Intensive Care Unit: Challenges in the Diagnosis and Treatment
}

\author{
Richa Gupta $^{1 *}$, Abida Malik ${ }^{1}$, Meher Rizvi ${ }^{1}$, Moied Ahmed ${ }^{2}$ \\ Department of ${ }^{1}$ Microbiology and ${ }^{2}$ Anaesthesiology, JNMC, Aligarh Muslim University, Aligarh, India.
}

\begin{tabular}{l} 
ARTICLE INFO \\
\hline Article history: \\
Received on: $06 / 07 / 2016$ \\
Revised on: $11 / 09 / 2016$ \\
Accepted on: $24 / 10 / 2016$ \\
Available online: $29 / 11 / 2016$ \\
\hline Key words: \\
Fungal infections, biofilm, \\
ICU, risk factors, mortality.
\end{tabular}

\begin{abstract}
Introduction: Fungal infections have risen exponentially and are a cause of significant morbidity and mortality in Intensive Care Unit. The study was done to assess the prevalence of fungal pathogens in ICU patients, their antifungal susceptibility profile and biofilm production.It also aimed at evaluating risk factors and outcome in patients infected with fungal pathogens.

Material and Methods: Samples were collected aseptically from 210 ICU patients from February 2012 to November 2014.They were cultured and identified by standard microbiological techniques. Antifungal susceptibility was performed according to CLSI guidelines.

Results: From 210 ICU patients, 52(24.7\%) were fungal pathogens. Majority of the fungal pathogens were Candida species 42(82.3\%) followed by Aspergillus fumigatus 10(19.2\%).Prevalence of Candida species was highest among urinary tract infections 20(39.2\%) while that of Aspergillus fumigatus among lower respiratory tract infections $9(17.6 \%) .34 .3 \%$ of C.albicans and 25\% C. dubliniensis were resistant to fluconazole. $40 \%$ of Aspergillus fumigatus were resistant to fluconazole and ketoconazole.21.8\% of C.albicans were resistant to nystatin. C. albicans $19(59.3 \%)$ followed by A. fumigatus $6(60 \%)$ were the most common biofilm producing fungal isolates. High degree of antibiotic resistance was exhibited by the biofilm film positive isolates compared with biofilm negative isolates. Patients with fungal infection had higher mortality rate(44.3\%) as compared to $40 \%$ in patients with bacterial infection.

Conclusion: Fungal infections are associated with a high mortality rate.This study confirms the importance of the epidemiological surveillance on fungal infections in the ICU setting for documenting species distribution and antifungal susceptibility patterns to guide therapeutic choices.
\end{abstract}

\section{INTRODUCTION}

Great advances in contemporary medicine and especially in critical care achieved during the last decades have contributed not only to longer survival of patients, but also to the increasing incidence of opportunistic infections caused by fungi. Complex medical and surgical problems, disruption of natural barriers, multiple invasive procedures and prolonged antibiotic treatment are some of the factors contributing to the alarming increase of fungal infections in the Intensive Care Unit (ICU) setting (Blot et al., 2008; Pfaller et al., 2007). In 2007 the results of EPIC II study including 1,265 ICUs in 75 countries revealed

* Corresponding Author

Dr. Richa Gupta, Department of Microbiology, JNMC, AMU, Aligarh, India.E-mail: sunita1457 @ gmail.com;Ph:07669170431 that $19 \%$ of pathogens isolated in ICU patients were fungi (Asmundsdottir et al., 2008). Candida species were predominantly isolated (17\%) followed by Aspergillus species. Fungal infections are associated with a high mortality and increased length of hospital stay and cost (Tragiannidis et al., 2013; Tabah et al., 2012). High attributable mortality may be due to delayed diagnosis and treatment, development of resistance or severity of illness. The purpose of the present review is to provide a practical approach to diagnosis and treatment of invasive fungal infections in the critically ill ICU patients.

Candidiasis is the leading cause of fungal infections in Intensive Care Unit (ICU) patients, with $C$. albicans being the most common causative agent (Blot et al., 2008; Pfaller et al., 2007).Even though Candida species is a part of the normal human flora, a small percentage can cause disease: 1) Candidaemia with 
or without endophthalmitis, 2) Disseminated haematogenous infections with deep organ involvement 3) Chronic disseminated candidiasis, most commonly found in haematological patients (Tragiannidis et al.,2013).

Candida bloodstream infections (BSIs) are a great proportion of nosocomial fungal infections and represent an important cause of morbidity and mortality in ICU patients (Tabah et al., 2012). Additionally, the true incidence of invasive candidiasis may be higher than estimated because of the small percentage of positive cultures obtained and the difficulty in making a diagnosis of invasive candidiasis without candidaemia (Alangaden,2011).There are several risk factors for the development of invasive disease such as the colonization of the gastrointestinal tract, disruption of the mucosa, neutropenia or immunosuppression, the increased use of medical procedures, and poor hygiene of the health care personnel (Van de Veerdonk et al., 2010).

Undoubtedly, the early diagnosis and treatment of invasive candidiasis is important but is often not an easy task because of the comorbidities and the delay in obtaining positive cultures. Candida colonization is referred to as a risk factor for developing invasive candidiasis (Dimopoulos et al., 2008). Several studies have presented the use of several clinical scores by using risk factors alone or in combination with sites of colonization in an attempt to identify patients at risk who might benefit from antifungal prophylaxis treatment. The widely used Candida Colonization Index (CCI) defined as the ratio of the number of culture positive surveillance sites for Candida spp. over the number of sites cultured. If the CCI is greater than 0.4 preemptive antifungal therapy should be commenced (Pittet et al., 1994). Additionally, a recent study presented the Candida Score (CS), which is based on the following risk factors: surgery upon admission, total parenteral nutrition, severe sepsis and multifocal colonization. A CS above 2.5 identifies high-risk patients who might benefit from antifungal prophylaxis treatment (Leon et al., 2009).

Aspergillus spp are moulds which are able to cause lifethreatening invasive disease in immunocompromised individuals and local disease in immunocompetent persons. The latter can present with a spectra ranging from localised infection of the lungs and sinuses to allergic reactions due to spore inhalation. The most commonly encountered include A. fumigatus followed by A. flavus and $A$. terreus. The epidemiology of aspergillosis in the ICU is difficult to establish due to the inhomogeneity of hospitalised patients, the diagnostic difficulties necessitating a biopsy and the difficulty in discriminating between colonisation and disease (Meersseman et al., 2007). Sometimes autopsy is necessary to prove the diagnosis (Meersseman et al., 2003) while a high mortality is reported (Dimopoulos et al., 2004). Possible sources of Aspergillus in the ICU include improperly cleaned ventilation systems, water systems, or even computer consoles (Warris et al., 2005). ICU patients with impaired immunity are prone to develop the invasive form of the disease in lungs and sinuses. Neutropenic patients usually develop the aggressive angioinvasive form while patients under steroid treatment present with a cavitating lesion. Anastomotic regions are the fungus target in patients with lung transplantation (Mehrad et al., 2001) while rarer presentations such as endocarditis or osteomyelitis have been described (Dimopoulos et al., 2010).

\section{MATERIAL AND METHODS}

The present study was carried out in the Department of Microbiology and ICU, Department of Anaesthesiology, J. N. Medical College, Aligarh Muslim University, Aligarh, during the period from February 2012 to November 2014.

A detailed clinical history was recorded from each patient. A complete general and systemic physical examination was also carried. Informed written consent was taken prior to any invasive procedure from all the patients and the investigations were performed after approval from Institutional Ethics Committee.

Various clinical specimens including tracheobronchial aspirate, pus, blood and urine from ICU patients were studied. The samples were obtained in duplicate and transported to the laboratory within 20 minutes of its collection. Identification of the microorganism and antifungal susceptibility tests were done according to the standard protocols.

For direct microscopic examination of fungal organisms, a $10 \% \mathrm{KOH}$ mount and Lacto-phenol Cotton Blue (LCB) mount was made.

The culture was done on two Sabouraud dextrose agar (SDA) slants containing chloramphenicol $(0.05 \mathrm{mg} / \mathrm{ml})$ by rolling over the surface and subsequently in BHI broth also. One tube was incubated at $25^{\circ} \mathrm{C}$ and the remaining tube and $\mathrm{BHI}$ broth were incubated at $37{ }^{\circ} \mathrm{C}$. After initial inoculation and incubation, tubes were examined daily for fungal growth upto 3 weeks.

The isolates were identified based on macroscopic and microscopic morphological characterstics following standard techniques described by Clinical Mycology by Anaissie et al., 2003,Chander et al., 2009 and Mackie and McCartney (2007).

The identification of yeast fungi was in accordance to i) colony characteristics, ii) Germ-tube test (GTT test), iii) growth at $42^{\circ} \mathrm{C}$, iv) morphology on CMA, v) Sugar fermentation tests and vii) Sugar assimilation tests (Clinical Mycology by Anaissie et al., 2003; Chander et al., 2009; Mackie and McCartney (2007).

The identification of mould fungi were in accordance to i) characteristic colony morphology on SDA, ii) by seeing the pigment on the back of SDA produced by fungal growth, iii) by making tease-mount preparation and identifying the morphology of coniodiophore and fungal head, iv) and in doubtful case, by using micro-slide culture technique and identifying the exact morphology of the hyphae, conidiophore and fungal heads.

Antifungal susceptibility of yeast isolates was done by broth micro dilution method proposed by NCCLS 2002, based on document no M-27A.Commercially available antimicrobial powders (Amphotericin B, nystatin, ketoconazole, flucytosine, fluconazole and itraconazole) were purchased from HiMedia 
Laboratories, Mumbai, India. ATCC 24433 Candida albicans was included each time a set of isolates was tested with each drugs.

Antifungal susceptibility of mould isolates was done by broth micro dilution method proposed by NCCLS 2002, based on document no M-38A.Commercially available antimicrobial powders (nystatin, amphotericin B, fluconazole and Itraconazole) were purchased from HiMedia Laboratories, Mumbai, India. Two QC organisms v.i.z A flavus, ATCC 204303 and A fumigatus ATCC 204305 were included each time a set of isolates was tested with each drugs. Sterility control was also performed by adding 1 $\mathrm{ml}$ un-inoculated drug free medium.

In vitro biofilm forming ability of the fungal isolates was tested by the tube method, as described by Mathur et al., 2006 with slight modification. $0.5 \mathrm{ml}\left(1.5 \times 10^{8}\right.$ organism $\left./ \mathrm{ml}\right)$ of 48 hour culture saline washed suspension was inoculated into a polysterene tube containing $4.5 \mathrm{ml}$ of trypticase soy broth (TSB) with $1 \%$ glucose (Mathur et al., 2006). Tubes were incubated at $37^{\circ} \mathrm{C}$ for 48 hours without agitation. After 48 hours, the culture broth in the tube was aspirated, and the tubes were washed twice with distilled water. The walls of tube were stained with $0.1 \%$ crystal violet after media and cells were discarded. Biofilm formation was considered positive when a visible film lined the wall and bottom of the tube. Ring formation at the liquid surface was not indicative of biofilm formation. Strong biofilm producer Staphylococcus epidermidis ATCC 35984 and nonbiofilm producer Candida albicans ATCC 10231 were used as positive and negative controls.

\section{RESULTS AND DISCUSSION}

Among 210 ICU patients, majority of the patients in the study group were males $121(64 \%)$ as compared to females 89 $(36 \%)$. Male is to female ratio was 1.4. From 210 ICU patients, $52(24.7 \%)$ fungal pathogens were isolated. The majority of fungal infections were observed in patients above 60 years of age. Full demography of our sample is illustrated in Table(1).

Table 1: Demographic profile of the ICU patients with Fungal infections.

\begin{tabular}{|c|c|}
\hline Patient's Characteristics & $\begin{array}{c}\text { Number of } \\
\text { Patients }(\%)\end{array}$ \\
\hline Age(years) $<20$ & $08(3.8)$ \\
\hline $21-40$ & $26(12.3)$ \\
\hline $41-60$ & $33(15.7)$ \\
\hline $61-80$ & $85(40.5)$ \\
\hline$>80$ & $58(27.6)$ \\
\hline Sex: male / female & $121 / 89$ \\
\hline \multicolumn{2}{|l|}{ Risk Factors } \\
\hline Endotracheal Tube/Tracheostomy & $126(60)$ \\
\hline Parentral Nutrition & $210(100)$ \\
\hline Central venous line & $84(40)$ \\
\hline Urinary Catheter & $210(100)$ \\
\hline Nasogastric Tube & $151(72)$ \\
\hline Mechanical Ventillation & $126(60)$ \\
\hline Comatosed $(\mathrm{GCS}<8)$ patients & $110(50)$ \\
\hline Prior antibiotic treatment taken & $198(94.3)$ \\
\hline \multicolumn{2}{|l|}{ Comorbid Conditions } \\
\hline Diabetes Mellitus with complications & $38(38.4)$ \\
\hline Surgery & $59(28.1)$ \\
\hline Chronic Renal Failure & $04(3.2)$ \\
\hline Tuberculosis & $04(3.2)$ \\
\hline
\end{tabular}

\begin{tabular}{|c|c|}
\hline Chronic Obstructive Pulmonary Disease & $16(12.8)$ \\
\hline Cancer & $10(11.2)$ \\
\hline Mortality rate & $93(44.3)$ \\
\hline ICU (length of stay) $<7 d$ & $35(16.8)$ \\
\hline $7-15 d$ & $111(52.8)$ \\
\hline$>15 d$ & $64(30.4)$ \\
\hline
\end{tabular}

Table 2: Antifungal sensitivity pattern of fungal isolates from ICU patients.

\begin{tabular}{llllll}
\hline $\begin{array}{l}\text { Name of the } \\
\text { Organism }\end{array}$ & \multicolumn{2}{l}{ Antifungal agents } \\
\cline { 2 - 6 } & Ket(\%) & Flu(\%) & Itr(\%) & $\operatorname{AmB(\% )}$ & Nt(\%) \\
\hline C.albicans $(\mathrm{n}=32)$ & Nil & $11(34.3)$ & Nil & Nil & $7(21.8)$ \\
C.dubliniensis $(\mathrm{n}=04)$ & Nil & $1(25)$ & Nil & Nil & Nil \\
C.glabrata $(\mathrm{n}=06)$ & Nil & $1(16.6)$ & Nil & Nil & Nil \\
A.fumigatus $(\mathrm{n}=10)$ & $4(40)$ & $4(40)$ & Nil & Nil & Nil \\
\hline
\end{tabular}

Table 3: Biofilm production in fungal isolates from ICU patients.

\begin{tabular}{lll}
\hline \multirow{2}{*}{ Name of ICU isolates } & \multicolumn{2}{c}{ Biofilm assay } \\
\cline { 2 - 3 } & Positive & Negative \\
\hline C. albicans $(\mathrm{n}=32)$ & $19(59.3)$ & $13(40.6)$ \\
C.dubliniensis $(\mathrm{n}=04)$ & $02(50)$ & $02(50)$ \\
C.glabrata ( $=06)$ & - & $06(100)$ \\
Aspergillus fumigatus $(\mathrm{n}=10)$ & $06(60)$ & $04(40)$ \\
\hline
\end{tabular}

Among fungal infections, 38(74.5\%) patients had pure fungal infection and remaining $13(25.4 \%)$ had mixed infection (with aerobic bacteria). Majority of the fungal pathogens were Candida species $42(80.7 \%)$ followed by Aspergillus fumigatus $10(19.2 \%)$. The most common pathogen isolated from patients was Candida albicans 32(61.5\%).

Candida albicans was most commonly isolated from urine samples $20(39.2 \%)$, followed by 6(11.7\%) from tracheal aspirate, $4(7.8 \%)$ from pus and $2(3.9 \%)$ from abdominal drains. $C$. glabrata was isolated from $4(7.8 \%)$ urine samples and $2(3.9 \%)$ pus samples. Aspergillus fumigatus was most commonly isolated from tracheal aspirate 9(17.6\%). 11(34.3\%) of C.albicans and $1(25 \%)$ C. dubliniensis were resistant to fluconazole. $4(40 \%)$ of Aspergillus fumigatus were resistant to fluconazole and ketoconazole. $7(21.8 \%)$ of C.albicans, were resistant to nystatin. All the fungal isolates were sensitive to itraconazole and amphotericin B. C. albicans strains were the most resistant against antimycotics, and C. glabrata seemed to be susceptible to nearly all antimycotic drugs. Patients with fungal infection had higher mortality rate $(44.3 \%)$ as compared to $40 \%$ in patients with bacterial infection. Antimycotic drug susceptibility comparison can be seen in Table (2)

The result of biofilm assay among fungal isolates is depicted in Table (3). C. albicans 19(59.3\%) followed by $A$. fumigatus $6(60 \%)$ were the most common biofilm producing fungal isolates. High degree of antibiotic resistance was exhibited by the biofilm film positive (BFP) isolates compared with biofilm negative isolates (BFN).

Patients in ICU are at a higher risk of acquiring nosocomial infections compared with patients in general wards 
due to the severity of the underlying illnesses and iatrogenic factors related to the high frequency of invasive procedures needed for the monitoring and treatment which include insertion of intravascular catheters, endotracheal intubation, and positive pressure ventilation, urinary catheterization and surgical operations. Studies from different parts of the world on nosocomial infections have shown that patient and treatment factors are risk factors for the development of nosocomial infections. These risk factors were also seen in our cases as elderly age (>60yrs); 143(56.2\%), Endotracheal Tube/Tracheostomy 126(60\%), Parentral Nutrition 210 (100\%), Central venous line $4(40 \%)$, Urinary Catheter 210(100\%), Nasogastric Tube 151(72\%), Mechanical Ventillation 126(60\%), Comatosed $(\mathrm{GCS}<8)$ patients $110(50 \%)$ and prior antibiotic treatment taken 192(91\%). Similar correlations were also observed in other studies as well (Ghiasian et al., 2014). Antibiotic therapy is one of the most important risk factors contributed to nosocomial fungal colonizations/infections by suppressing endogenous bacterial flora and decrease of nonbacterial emerging flora, mainly in colorectal tract and in areas close to the ureteral meatus (Alvarez-Lerma et $a l ., 2003)$. In the current study, antibiotic therapy was the principal (94.3\%) predisposing factor and a total of 198 patients (out of 210) used broad-spectrum antibiotics. In support of our study, other researchers from different countries concluded that broadspectrum antibiotic therapy can be one of the most important predisposing factors for development of nosocomial fungal infections in patients admitted to ICUs (Zarei et al., 2012, Delgado et al., 2010). Nonetheless, some other studies found greater prevalence of this factor and showed that all critically ill patients with nosocomial fungal infections had received antibiotics (Passos et al., 2005). According to Sydnor and Perl, admission to ICU itself was a significant risk factor; roughly $25 \%$ of nosocomial infections occur in intensive care units (ICUs), which have been estimated to increase ICU length of stay by 4.3 to 15.6 days (Sydnor et al., 2011). Length of stay in the ICU is also associated with increased risk for fungal infections, which rises rapidly after 7-10 days (M'ean et al., 2008). In our study also maximum no. of patients had hospital stay of 7-15 days.

In this study, we have identified $C$. albicans, $C$. glabrata, C. dubliniensis and Aspergillus fumigatus to be the most important pathogens. The most common pathogen isolated from patients was Candida albicans 32(61.5\%). There are many reports that support our finding of C.albicans being the most common pathogen over non-albicans candida species (Francuzik et al., 2015, Rajeswari et al., 2012). In contrast to our study, Mujika et al., 2004 and Shin et al., 2002 showed a trend towards an increasing prevalence of infections caused by species of non-albicans Candida.

We observed a higher incidence of $C$. glabrata infections in patients suffering from diabetes mellitus. Such findings have also been reported by Segi reddy et al., 2009. It is known that $C$. glabrata has lower virulence compared to $C$. albicans. This fact may explain why humans lack specific host defense mechanisms against this commensal microorganism. This fungus, once acquired, may be carried asymptomatically over a prolonged period of time. Diabetic patients have an impaired immune system; hence they are more susceptible to $C$. glabrata infections, contrary to a resistant, healthy population (Trick et al., 2002).

In ICU patients, the most common types of Candida infections are seen to comprise bloodstream, catheter-related, intra-abdominal, and urinary tract infections (Eggimann et al., 2003 ). In our patients, culture isolations were obtained in $47 \%$ of urine samples followed by $29.4 \%$ of tracheal aspirate in addition to a varying percentage from other samples like pus $(11.7 \%)$ and abdominal drains samples (3.9\%). Similar findings were observed by Kaur et al., 2014 who observed culture isolations were obtained in $39.8 \%$ of urine samples followed by $31.06 \%$ of tracheal aspirate in addition to a varying percentage from other samples like blood $(8.7 \%)$, endotracheal tube $(6.7 \%)$, and abdominal drains samples (2.9\%). 11(34.3\%) of C.albicans and 1(25\%) C. dubliniensis were resistant to fluconazole. 4(40\%) of Aspergillus fumigatus were resistant to fluconazole and ketoconazole. 7(21.8\%) of C.albicans, were resistant to nystatin. All the fungal isolates were sensitive to itraconazole and amphotericin B. C. albicans strains were the most resistant against antimycotics, and $C$. glabrata seemed to be susceptible to nearly all antimycotic drugs. Other studies (Rajeshwari et al., 2012, Kaur et al., 2014) reported higher rates of antifungal resistance. Rajeshwari et al., 2012 reported that itraconazole had highly resistance activity (98.2\%), second most Nystatin (83.9\%) followed by Clotrimazole (75\%), Amphoteracin B $(67.85 \%)$ and Ketoconazole $(66.0 \%)$. The lowest antibiotic resistance was observed in Fluconazole (57.14\%).

The ability to form biofilms is associated with the pathogenicity and as such should be considered as an important virulence determinant during fungal infections. Biofilms may help maintain the role of fungi as commensals and pathogen, by evading host immune mechanisms, resisting antifungal treatment, and withstanding the competitive pressure from other organisms. Consequently, biofilm related infections are difficult to treat. The biofilm production is also associated with high level of antimicrobial resistance of the associated organisms (Mohandas et al., 2011). In the present study fungal isolates were studied for their ability to produce biofilm. Biofilm production was more in Candida albicans than other species. Similar findings were observed by Rajeshwari et al., 2012. C. albicans 19(60.4\%) followed by $A$. fumigatus $06(60 \%)$ were the most common biofilm producing fungal isolates. However, Shin et al., 2002 and Pathak et al., 2012 reported higher biofilm-forming ability of NAC spp. than $C$. albicans species All the antifungal resistant strains were biofilm producing fungal isolates. As predicted by earlier work (Silva et al., 2009), strongly biofilm isolates had highest antibiotic resistance. There were no significant differences in biofilm production when grouping the strains according to the patients's age, and site of infection. The understanding of microbial biofilm structure and the use of modern technology to bring about modification of the medical devices will lead to decreased microbial infection of medical devices. 


\section{CONCLUSION}

The results showed that prevalence of fungal nosocomial infections is increasing in ICU patients. The main predisposing factors are antibiotic and corticotherapy, urinary catheterization, and extended hospitalization. The incidence of nosocomial UTIs can be decreased in ICU patients by shortening the duration of urinary catheterization, avoiding extra antibiotics and corticosteroids prescription, and finally, controlling the predisposing factors and underlying conditions. The results obtained here open perspectives of early investigations that are aimed at establishing and broadening knowledge of understanding relationship between strain types and properties such as pathogenicity, commensality, and infectivity, an important interplay in host-pathogen relationship specially as far as fungal infections are concerned.

\section{Financial support and sponsorship: Nil}

Conflict of Interests: There are no conflicts of interest.

\section{REFERENCES}

Alangaden GJ. Nosocomial fungal infections: epidemiology, infection control, and prevention. Infect Dis Clin North Am. 2011; 25: 201-25.

Alvarez-Lerma F, Nolla-Salas J, Leon C, Palomar M, Jorda R, Carrasco N.Candiduria in critically ill patients admitted to intensive care medical units.Intensive Care Med. 2003;29:1069-76.

Anaissie EJ,McGinnis MR, Pfaller MA. 2003.Clinical mycology. Philadelphia (PA): Churchill Livingstone.

Asmundsdottir LR, Erlendsdottir H, Haraldsson G, Guo H, Xu J, Gottfredsson M. Molecular epidemiology of candidemia: evidence of clusters of smoldering nosocomial infections. Clin Infect Dis. 2008; 47: $17-24$.

Blot S, Dimopoulos G, Rello J, Vogelaers D. Is Candida really a threat in the ICU? Curr Opin Crit Care. 2008;14:600-4.

Chander J. 2009. Textbook of medical mycology: Aspergillosis. 3rd edition; 24: 99-102.

Delgado J, Calvo N, Gomis A, Perez-Flores I, Rodriguez A, Ridao N, et al. Candiduria in renal transplant recipients: incidence, clinical repercussion, and treatment indication. Transplant Proc. 2010;42:2944-6.

Dimopoulos G, Ntziora F, Rachiotis G, Armaganidis A, Falagas ME. Candida albicans versus non-albicans intensive care unit-acquired bloodstream infections: differences in risk factors and outcome. Anesth Analg. 2008;106:523-9.

Dimopoulos G, Piagnerelli M, Berre J, Eddafali B, Salmon I, Vincent JL. Disseminated aspergillosis in intensive care unit patients: An autopsy study. J. Chemother. 2003; 15:71-75.

Dimopoulos G, Tsangaris I, Poulakou G, Panayiotides J, Tsaknis G,Orfanos S, Armaganides A. Post-operative Aspergillus mediastinitis in a man who was immunocompetent: A case report. J. Med. Case Rep. 2010; 4:312.

Eggimann P, Garbino J, and Pittet D.Management of Candida species infections in critically ill patients. The Lancet Infectious Diseases. $2003 ; 3: 772-785$.

Francuzik W, Lodowska A, Adamska K, Adamski Z, Mikstacki A and Tamowicz B. Prevalence of Yeast Fungal Infections in Intensive Care Unit in Poland. 2015.

Ghiasian SA, Aghamirian MR, Eshghi GR.Nosocomial Candiduria in Critically Ill Patients Admitted to Intensive Care Units in Qazvin, Iran. Avicenna J Clin Microb Infec. 2014; 12: 21622.

Kaur R, Ritu G, Dhakad M S, Bhalla P,and Kumar R.Epidemiology and Virulence Determinants including Biofilm Profile of Candida Infections in an ICU in a Tertiary Hospital in India. Hindawi
Publishing Corporation Journal of Mycology.2014, Article ID 303491, 8 pages.

Leon C, Ruiz-Santana S, Saavedra P, Galvan B, Blanco A, Castro C, et al. Usefulness of the "Candida score" for discriminating between Candida colonization and invasive candidiasis in non-neutropenic critically ill patients: a prospective multicenter study. Crit Care Med. 2009;37:1624-33.

M'ean M, Marchetti O, and Calandra T.Bench-to-bedside review: Candida infections in the intensive care unit. Critical Care.2008; 12:204.

Mackie and McCartney. 2007. Practical Medical Microbiology. Fourteenth Edition. Published by Churchill Livinstone.

Mathur T, Singhal S, Khan S, Upadhyay DJ, Fatma T, Rattan A. Detection of biofilm formation among the clinical isolates of Staphylococci: evaluation of three different screening methods. Indian J Med Microbiol. 2006; 24:2-9.

Meersseman W, Lagrou K, Maertens J, Wijngaerden VE. Invasive aspergillosis in the intensive care unit. Clin. Infect. Dis. 2007; 45:205-216.

Meersseman W,Vandecasteele SJ, Wilmer A,Verbeken E, Peetermans WE, Wijngaerden VE. Invasive aspergillosis in critically ill patients without malignancy. Am. J. Resp. Crit. Care Med. 2004; 170:621625 .

Mehrad B, Paciocco G, Martinez FJ, Ojo TC, Iannettoni MD, Lynch JP. Spectrum of Aspergillus infection in lung transplant recipients: Case series and review of the literature. Chest. 2001; 119: 169-175.

Mohandas V, Ballal M. Distribution of Candida Species in different clinical samples and their virulence: Biofilm formation, proteinase and phospholipase production: A study on hospitalized patients in Southern India.2011; 3: 4-8.

Mujika MT, Finquelievich JL, Jewtuchowicz V, Iovannitti CA. Prevalence of Candida albicans and Candida non-albicans in clinical samples during 1999-2001. Rev Argent Microbiol. 2004; 36:107-12.

Passos XS, Sales WS, Maciel PJ, Costa CR, Miranda KC, Lemos Jde A.Candida colonization in intensive care unit patients' urine. Mem Inst Oswaldo Cruz. 2005; 100:925-8.

Pathak A K, Sharma S, and Shrivastva P.Multi-species biofilm of Candida albicans and non-Candida albicans Candida species on acrylic substrate. Journal of Applied Oral Science.2012;20:70-75.

Pfaller MA, Diekema DJ. Epidemiology of invasive candidiasis: a persistent public health problem. Clin Microbiol Rev.2007;20:133-63.

Pittet D, Monod M, Suter PM, Frenk E, Auckenthaler R. Candida colonization and subsequent infections in critically ill surgical patients. Ann Surg. 1994;220:751-8.

Rajeswari P, Vijayalakshmi P, Jegadeeshkumar D. Prevalence Of Biofilm Producing MDR Candida Albicans And Non Candida Albicans Isolate From Medical Devices. International Journal of Applied Biology and Pharmaceutical Technology. 2012;3:254-263.

Segireddy M, Johnson LB, Szpunar SM, Khatib R. Differences in patient risk factors and source of candidaemia caused by Candida albicans and Candida glabrata. Mycoses. 2011;54: 39-43.

Shin JH, Kee SJ, Shin MG, Kim SH, Shin DH, Lee SK, Suh SP, RyangDW. Biofilm production by isolates of Candida species recovered from nonneutropenic patients: comparison of bloodstream isolates with isolates from other sources. J. Clin. Microbiol.2002; 40: 1244-1248.

Silva S, Henriques M, Martins A, Oliveria R, Williams D, Azeredo J.Biofilms of non-candida albicans and Candida species: quantification, structure and matrix composition. Med. Mycol.2009; 47:681-689.

Sydnor E R M and Perl T M.Hospital epidemiology and infection control in acute-care settings.Clinical Microbiology Reviews.2011.24:141-173.

Tabah A, Koulenti D, Laupland K, Misset B, Valles J, Bruzzi De Carvalho F, et al. Characteristics and determinants of outcome of hospital-acquired bloodstream infections in intensive care units: the EUROBACT International Cohort Study. Intensive Care Med. 2012; 38: 1930-45.

Tragiannidis A, Tsoulas C, Kerl K, Groll AH. Invasive candidiasis: update on current pharmacotherapy options and future 
perspectives. Expert Opin Pharmacother. 2013;14:15-28.

Trick WE, Fridkin SK, Edwards JR, Hajjeh RA, Gaynes RP. Secular trend of hospital-acquired candidemia among intensive care unit patients in the United States during 1989-1999. Clinical infectious diseases.2002;35:627-30

Van de Veerdonk FL, Kullberg BJ, Netea MG. Pathogenesis of invasive candidiasis. Curr Opin Crit Care. 2010;16:453-9.

Warris A, Verweij PE. Clinical implications of environmental sources for Aspergillus. Med. Mycol. 2005;43:59-65.

Zarei MA, Zarrin M, Ghanatir F, Vazirianzadeh B. Candiduria in hospitalized patients in teaching hospitals of Ahvaz. Iran J Microbiol. 2012;4:198-203.

\section{How to cite this article:}

Gupta R, Malik A, Rizvi M, Ahmed M. An Alarming Increase of Fungal Infections in Intensive Care Unit: Challenges in the Diagnosis and Treatment. J App Pharm Sci, 2016; 6 (11): 114-119. 